TRANSACTIONS OF THE

AMERICAN MATHEMATICAL SOCIETY

Volume 355, Number 7 , Pages 2991-3008

S 0002-9947(03)03279-3

Article electronically published on March 17, 2003

\title{
STABILITY OF SMALL AMPLITUDE BOUNDARY LAYERS FOR MIXED HYPERBOLIC-PARABOLIC SYSTEMS
}

\author{
F. ROUSSET
}

\begin{abstract}
We consider an initial boundary value problem for a symmetrizable mixed hyperbolic-parabolic system of conservation laws with a small viscosity $\varepsilon, u_{t}^{\varepsilon}+F\left(u^{\varepsilon}\right)_{x}=\varepsilon\left(B\left(u^{\varepsilon}\right) u_{x}^{\varepsilon}\right)_{x}$. When the boundary is noncharacteristic for both the viscous and the inviscid system, and the boundary condition dissipative, we show that $u^{\varepsilon}$ converges to a solution of the inviscid system before the formation of shocks if the amplitude of the boundary layer is sufficiently small. This generalizes previous results obtained for $B$ invertible and the linear study of Serre and Zumbrun obtained for a pure Dirichlet's boundary condition.
\end{abstract}

\section{INTRODUCTION}

We consider a one-dimensional system of conservation laws with a small parameter $\varepsilon$ set in the domain $x>0$,

$$
u_{t}^{\varepsilon}+F\left(u^{\varepsilon}\right)_{x}=\varepsilon\left(B\left(u^{\varepsilon}\right) u_{x}^{\varepsilon}\right)_{x}, x>0, t>0,
$$

where $u^{\varepsilon} \in \mathbb{R}^{n}$ and $F: \mathcal{U} \rightarrow \mathbb{R}^{n}, B: \mathcal{U} \rightarrow \mathbb{R}^{n \times n}$. We will assume that $F$ and $B$ are smooth $\left(\mathcal{C}^{\infty}\right)$. We add to this system an initial condition $u^{\varepsilon}(0, x)=u_{0}(x)$ and a boundary condition that we will detail later. We assume that the eigenvalues of $B$ have nonnegative real part and that the rank of $B$ does not depend on $u$. We will denote it by $r, 1 \leq r \leq n$. Note that $B$ is not necessarily invertible. We are interested in the limit of $u^{\varepsilon}$ when $\varepsilon$ tends to zero. We expect that $u^{\varepsilon}$ tends to a solution of the inviscid problem:

$$
u_{t}+F(u)_{x}=0
$$

with some boundary conditions to be determined. At first we make the natural assumptions to ensure the well-posedness of the Cauchy problem for (11) [6]. There exists a change of variable $u \rightarrow v(u)$ with inverse $u=g(v)$ in which the system can be rewritten as

$$
g(v)_{t}+f(v)_{x}=\varepsilon\left(b(v) v_{x}\right)_{x}
$$

with the following properties:

- (H1) $b(v)$ is block diagonal,

$$
b(v)=\left(\begin{array}{cc}
0 & 0 \\
0 & b_{1}(v)
\end{array}\right),
$$

with $b_{1}(v) \in G L_{r}(\mathbb{R})$.

Received by the editors January 30, 2002 and, in revised form, December 13, 2002.

2000 Mathematics Subject Classification. 35K50, 35L50, 35L65, 76H20.

(C)2003 American Mathematical Society 
- (H2) $d g(v)$ is lower block diagonal,

$$
d g(v)=\left(\begin{array}{cc}
v & 0 \\
\cdot & \tilde{g}(v)
\end{array}\right),
$$

with $\tilde{g}(v) \in G L_{r}(\mathbb{R})$.

By analogy to the terminology in gas dynamics, we shall refer to $v$ as the primitive variable.

Next we assume that (1) is symmetrizable mixed hyperbolic-parabolic:

- (H3) there exists a positive definite symmetric $\Sigma(u)$ such that

(1) $\Sigma(u) d F(u)$ is symmetric,

(2) $\Sigma(u) B(u) X \cdot X \geq \alpha|B(u) X|^{2}, \forall X \in \mathbb{R}^{n}$,

where $\alpha>0$, and $\cdot$ stands for the scalar product of $\mathbb{R}^{n}$.

We denote by $v=(w, z)$ the corresponding block decomposition of $v$.

Note that since $d f(v)=d F(u) d g(v), b(v)=B(u) d g(v)$, setting $S(v)=d g(v)^{t} \Sigma(v)$, we get that (H3) is equivalent to

- $\left(\mathbf{H 3}^{\prime}\right)$ There exists $S(v)$ such that

(1) $S(v) d g(v)$ is positive definite symmetric,

(2) $S(v) d f(v)$ is symmetric,

(3) $S(v) b(v) X \cdot X \geq \alpha|z|^{2}, \forall X=\left(\begin{array}{c}w \\ z\end{array}\right) \in \mathbb{R}^{n}$.

We point out that $(\mathrm{H} 3)(1)$ implies that the inviscid system (2) is hyperbolic. Moreover (H3)(2) implies that $d g^{t} \Sigma d g$ is block diagonal (see [11, Lemma 4.1). Hence thanks to (H2), we get

$$
S=\left(\begin{array}{cc}
S_{w}(v) & 0 \\
* & S_{z}(v)
\end{array}\right)
$$

where $S_{w}(v)$ is positive definite symmetric. Consequently, writing the block decomposition of $d f$ as

$$
d f(v)=\left(\begin{array}{cc}
h(v) & * \\
* & *
\end{array}\right),
$$

we get from $\left(\mathrm{H}^{\prime}\right)(2)$ that $S_{w}(v) h(v)$ is symmetric. This means that the system obtained from (3) by removing the second equation is symmetric-hyperbolic.

Finally, we also assume that the hyperbolic and parabolic modes do couple:

- (H4) The kernel of $B$ does not contain any eigenvector of $d F$.

The structural hypotheses (H1-H4) are verified by many physical equations as those of compressible gas dynamics and magnetohydrodynamics.

Next we make hypotheses to deal with the initial boundary value problem. We focus on the case of a noncharacteristic boundary. We assume that the boundary is noncharacteristic for both the viscous (11) and the inviscid (2) systems:

- (H5) $d F(u)$ and $h(v)$ are nonsingular.

Note that an inflow or outflow boundary condition makes the boundary noncharacteristic in most cases for the Euler and Navier-Stokes equations. These boundary conditions have a physical meaning since they appear in problems with aperture, such as in oil recovery. The analysis of an impermeable boundary would be different since in this case the boundary is characteristic.

We denote by $q$ the number of eigenvalues of positive real part of $d F(U)$, and by $p$ the number of eigenvalues of positive real part of $h(v)$. An initial boundary 
value problem for (11) needs $p+r$ scalar independent boundary conditions, and an initial boundary value problem for (2) needs $q$ independent scalar boundary conditions. We deal with boundary conditions for (3) that are linear with respect to the primitive variable $v$. We write the boundary condition for (3) as

$$
L v(t, 0)=\left(\begin{array}{l}
l w \\
z
\end{array}\right)(t, 0)=g
$$

where $l$ is a linear map that has rank $p$ and $g$ is a given constant.

In the following, in order to make energy estimates, we assume that the boundary condition (5) is "dissipative":

- (H6) $\exists \beta>0, \forall v \in \mathcal{V}, \forall X=\left(\begin{array}{c}X_{1} \\ X_{2}\end{array}\right)$, such that $L v=g, L X=0$, and we have

$$
S_{w}(v) h(v) X_{1} \cdot X_{1} \leq-\beta\left|X_{1}\right|^{2} .
$$

There are physical boundary conditions in the form (5) that satisfy (H6). The case of the isentropic gas dynamics will be studied below.

Note that thanks to hypotheses (H1-H5), we have $p \leq q \leq p+r$ ([12], Corollary 1 ). Hence in the case $q<p+r$, there is a loss of boundary condition when $\varepsilon$ tends to zero. It is due to a fast change of $u^{\varepsilon}$ in a vicinity of the boundary: the boundary layer. In the noncharacteristic case the size of the boundary layer is $\varepsilon$. When $\varepsilon$ tends to zero, the expected behaviour of $u^{\varepsilon}$ is ([2], [1], [12])

$$
u^{\varepsilon} \sim u^{i n t}(t, x)+U\left(t, \frac{x}{\varepsilon}\right),
$$

where $u^{\text {int }}$ is a solution of (2) with the initial condition $u^{\text {int }}(0, x)=u_{0}(x)$ and some boundary conditions that we have to determine. $U(t, z)$ is a boundary layer; it is a solution of a differential problem where the time is only a parameter:

$$
\begin{aligned}
& B(U) U^{\prime}=F\left(U+u^{i n t}(t, 0)\right)-F\left(u^{i n t}(t, 0)\right), \\
& U(t,+\infty)=0, \\
& L v\left(U(t, 0)+u^{i n t}(t, 0)\right)=g .
\end{aligned}
$$

Note that when $r<n$, we have an algebraic differential system. This problem has solutions if and only if $u^{i n t}(t, 0)$ belongs to the subset $\mathcal{C}$, where

$$
\mathcal{C}=\left\{U_{+} \in \mathbb{R}^{n}, \exists U,\left\{\begin{array}{l}
B(U) U^{\prime}=F\left(U+U_{+}\right)-F\left(U_{+}\right), \\
U(+\infty)=0, \\
L v\left(U(0)+U_{+}\right)=g .
\end{array}\right\}\right.
$$

This set $\mathcal{C}$ is called the set of residual boundary conditions. It was studied in the case $r=n$ in [2], 4] and in the general case in [1]. Assuming that $u_{0}(0)$ satisfies the boundary condition (5), i.e.,

$$
L v\left(u_{0}(0)\right)=0,
$$

we have $u_{0}(0) \in \mathcal{C}$ (the associated profile of the boundary layer is $U=0$ ). Moreover, thanks to $(\mathrm{H} 1-\mathrm{H} 6)$ we can use 11], Lemma 4.2 and Theorem 1.1. $\mathcal{C}$ is a smooth submanifold in the vicinity of $u_{0}(0)$ that has dimension $q$ and that is transverse to the unstable subspace of $d F\left(u_{0}(0)\right)$. Consequently, thanks to a theorem of [8], there exists a continuous solution of (2) with the boundary condition $u^{i n t}(t, 0) \in \mathcal{C}$ defined on $[0, T]$ for some positive small time $T$. Assuming some higher-order compatibilities between $u_{0}(0)$ and $\mathcal{C}$, we can even get a smooth solution $u$. Using 
the same method as in [4, we can show the existence of an approximate solution of (11) in the form

$$
u^{a p p}(t, x)=u^{i n t}(t, x)+U\left(t, \frac{x}{\varepsilon}\right)+\sum_{i=1}^{M} \varepsilon^{i}\left(u^{i n t, i}(t, x)+U^{i}\left(t, \frac{x}{\varepsilon}\right)\right)
$$

such that

$$
\begin{gathered}
L v\left(u^{a p p}(t, 0)\right)=0, \quad u^{a p p}(0, x)=u_{0}(x), \\
\partial_{t} u^{a p p}+\partial_{x} F\left(u^{a p p}\right)-\varepsilon \partial_{x}\left(B\left(u^{a p p}\right) \partial_{x} u^{a p p}\right)=R^{\varepsilon}
\end{gathered}
$$

where

$$
\left\|R^{\varepsilon}\right\|_{L^{\infty}[0, T], L^{2}} \leq C \varepsilon^{M} .
$$

Our aim is to show that the true solution $u^{\varepsilon}$ is close to the approximate solution if the boundary layer is sufficiently weak. More precisely,

Theorem 1 (Nonlinear stability). Assuming (H1-H6) and that $u_{0} \in H^{7}\left(\mathbb{R}_{+}\right)$, there exists $\delta>0$ such that if

$$
\sup _{t \in[0, T]}\left(\left|\partial_{z} U(t, \cdot)\right|_{\infty}+\int_{0}^{+\infty} z\left|\partial_{z} U(t, z)\right| d z+\int_{0}^{+\infty}\left|\partial_{z} U(t, z)\right| d z\right) \leq \delta,
$$

then

$$
u^{\varepsilon}-u^{i n t} \rightarrow 0
$$

when $\varepsilon \rightarrow 0$ in $L^{\infty}\left([0, T], L^{2}\right)$.

To prove this theorem, we actually need to start from a very accurate approximate solution $u^{a p p}$. Indeed, we will take $M=3$ in the expansion (8). The construction of such a high-order expansion requires a lot of regularity on $u^{\text {int }}$ (see [4]). This is why we have to assume so much regularity on $u_{0}$. We actually get a more precise estimate:

$\left\|\left(u^{\varepsilon}-u^{a p p}\right)(t, \cdot)\right\|_{L^{2}}^{2}+\varepsilon^{2}\left\|\partial_{x}\left(u^{\varepsilon}-u^{a p p}\right)(t, \cdot)\right\|_{L^{2}}^{2}+\varepsilon^{5}\left\|\left(u^{\varepsilon}-u^{a p p}\right)(t, \cdot)\right\|_{W^{1, \infty}}^{2} \leq C \varepsilon^{6}$.

Our method can also provide estimates in $L^{\infty}\left([0, T], H^{s}\right)$ for any $s$.

The proof of Theorem 1 relies on energy estimates. We use the primitive variable $v$; hence we work on the form (B) of the equation. We combine the energy estimate of the totally parabolic case $(r=n)$ [2], [4] with an energy estimate of Kawashima's type [6] and a careful study of the boundary values. A key argument of the proof is the following lemma of [13]:

Lemma 2 (S-K [13]). Assuming (H1-H4), there exists a skew-symmetric K(u) and a positive constant $\theta$ such that

$$
\left((K(u) d F(u))^{s}+\Sigma(u) B(u)\right) X \cdot X \geq \theta|X|^{2}, \quad \forall X \in \mathbb{R}^{n}, \forall u \in \mathcal{U}
$$

where $(K d F)^{s}=\frac{1}{2}\left(K d F+(K d F)^{t}\right)$.

Note that setting $k(v)=d g(v)^{t} K(g(v))$ we can rewrite this result as

$$
\left((k(v) d f(v))^{s}+S(v) b(v)\right) X \cdot X \geq \theta|X|^{2}, \quad \forall X \in \mathbb{R}^{n}
$$

with

$$
k(v) d g(v) \text { skew-symmetric. }
$$


In [9], Lemma 2 and the estimates of [6] combined with pointwise Green's functions bounds were already used to prove the nonlinear asymptotic stability of weak time-independent viscous shock profiles for (11). The asymptotic stability of a timeindependent profile of the boundary layer together with the stability of other nonlinear waves was studied in 10 for the isentropic gas dynamics rewritten as a $p$ system in Lagrangian coordinates.

Let us give an example of an application of our theorem. Consider the isentropic gas dynamics where $v=(\rho, \mathrm{v}), \rho$ being the mass density and $\mathrm{v}$ the fluid velocity,

$$
g(v)=\left(\begin{array}{l}
\rho \\
\rho \mathrm{v}
\end{array}\right), \quad f(v)=\left(\begin{array}{l}
\rho \mathrm{v} \\
\rho \mathrm{v}^{2}+p(\rho)
\end{array}\right), \quad b(v)=\left(\begin{array}{cc}
0 & 0 \\
0 & \nu(\rho)
\end{array}\right) .
$$

Here we assume that $\nu>0$ and that $p^{\prime}>0$ (hyperbolicity). The sound speed is $c(\rho)=\sqrt{p^{\prime}(\rho)}$. (H1-H4) are verified; moreover, the eigenvalues of $d F$ are $\mathrm{v} \pm c$ and the eigenvalue of (the $1 \times 1$ matrix) $h$ is $\mathrm{v}$.

Let us first consider an outflow boundary condition

$$
\mathrm{v}(t, 0)=\mathrm{v}^{-}
$$

with $\mathrm{v}^{-}<0$. In this case, $l=0$, and $(\mathrm{H} 6)$ becomes

$$
\mathrm{v}^{-}|X|^{2} \leq-\beta|X|^{2}
$$

hence, it is satisfied. The compatibility condition (7) becomes $\mathrm{v}_{0}(0)=\mathrm{v}_{-}$. It suffices to impose $\mathrm{v}_{0}(0)+c\left(\rho_{0}(0)\right) \neq 0$ to get $(\mathrm{H} 5)$.

If we consider an outflow boundary condition

$$
\mathrm{v}(t, 0)=\mathrm{v}_{-}, \quad \rho(t, 0)=\rho_{-},
$$

where $\mathrm{v}_{-}>0$, we have $l=I d$, Ker $l=\{0\}$, and hence (H6) is true. The compatibility condition becomes $\rho_{0}(0)=\rho_{-}, \mathrm{v}_{0}(0)=\mathrm{v}_{-}$and hence we get (H7) if $\mathrm{v}_{-}-c\left(\rho_{-}\right) \neq 0$. Moreover, in the case $\mathrm{v}^{-}-c\left(\rho_{-}\right)>0$, we have $q=p+r=2$; hence there is no boundary layer and the hypothesis (9) is always satisfied.

For a more general discussion of the various boundary conditions for the nonisentropic gas dynamics, we refer to 12 .

As in the totally parabolic case $r=n$, the smallness assumption (9) in Theorem 1 is linked with the stability of the boundary layer. In [12] an example of a large unstable boundary layer is given. To understand the mechanism of instability in the boundary layer, we set $\theta=\frac{t}{\varepsilon}, z=\frac{x}{\varepsilon}$, we fix some time $\tau$ in $u^{a p p}$ and we linearize about the leading term of $u^{a p p}$ with respect to $\varepsilon$. We get the linear system

$$
\begin{aligned}
& \partial_{\theta} u=\mathcal{L}_{\tau} u, \\
& L d v\left(u^{i n t}(\tau, 0)+U(\tau, 0)\right) u(\theta, 0)=0, \\
& u(0, z)=u_{0}(z)
\end{aligned}
$$

where

$$
\begin{aligned}
\mathcal{L}_{\tau} u= & \left(b\left(u^{i n t}(\tau, 0)+U(\tau, z)\right) u^{\prime}+d B\left(u^{i n t}(\tau, 0)+U(\tau, z)\right) u U^{\prime}(\tau, z)\right. \\
& \left.-d F\left(u^{i n t}(\tau, 0)+U(\tau, z)\right) u\right)^{\prime} .
\end{aligned}
$$

Here' stands for $\frac{\partial}{\partial z}$. We will say that the profile of the boundary layer $u^{\text {int }}(\tau, 0)+$ $U(\tau, z)$ for some fixed $\tau$ is linearly stable if the solutions of this system tend to zero 
when $t$ tends to $+\infty$. The linear stability is linked with the spectral stability as was shown in [15], [9], [14]. Let us define the domain of $\mathcal{L}_{\tau}$ as

$$
\mathcal{D}\left(\mathcal{L}_{\tau}\right)=\left\{u=d g(V(\tau, z)) v, v=\left(\begin{array}{c}
w \\
z
\end{array}\right), w \in H^{1}\left(\mathbb{R}_{+}\right), z \in H^{2}\left(\mathbb{R}_{+}\right), L v(t, 0)=0\right\}
$$

where $V(\tau, z)$ is defined by $g(V(\tau, z))=u^{i n t}(\tau, 0)+U(\tau, z)$.

In [12], it is shown that the essential spectrum of $\mathcal{L}_{\tau}$ is confined in $\{\mathcal{R} e \lambda<$ $0\} \cup\{0\}$ thanks to $(\mathrm{H} 1-\mathrm{H} 5)$. In the unstable half-plane $\{\mathcal{R} e \lambda \geq 0\} \backslash\{0\}$ the spectrum only consists of eigenvalues. Consequently, a necessary condition for the linear stability of the boundary layer is that the operator $\mathcal{L}_{\tau}$ does not have eigenvalues in the unstable half-plane $\{\mathcal{R} e \lambda \geq 0\}$ (spectral stability). An Evans function machinery was developed in [12] to find sufficient conditions of instability.

In the first part, we show that spectral stability holds for weak boundary layers.

Theorem 3 (Spectral stability). There exists $\delta>0$ such that, assuming (H1-H6) and

$$
\left|\partial_{z} U(\tau, \cdot)\right|_{\infty}+\int_{0}^{+\infty} z\left|\partial_{z} U(\tau, z)\right|+\int_{0}^{+\infty}\left|\partial_{z} U(\tau, z)\right| d z \leq \delta,
$$

then $\mathcal{L}_{\tau}$ does not have eigenvalues in the unstable half-plane $\{\mathcal{R} e \lambda \geq 0\}$.

The proof also relies on energy estimates. We first give a direct proof of Theorem 3 because it seems more enlightening to present the main ingredients of the proof of Theorem 1 in the simpler linear time-independent setting of Theorem 3. This result is not used in the proof of Theorem 1. The result of Theorem 3 could be deduced from direct energy estimates on the time evolutionary sytem (12), (13), (14). Nevertheless it is interesting to study the spectral stability since we can expect that, as in the totally parabolic case, the sharp assumption of spectral stability implies the nonlinear convergence result [5].

Note that our result of Theorem 3 (obtained by a different method) implies the result of the appendix of [12] where only Dirichlet's boundary conditions were considered for (1).

In the second part, we give the proof of the full nonlinear stability result of Theorem 1.

\section{Spectral stability}

In this section, we prove Theorem 3. We study the eigenvalue problem

$$
\begin{aligned}
& \lambda u-\mathcal{L}_{\tau} u=0, \\
& L d v\left(u^{i n t}(\tau, 0)+U(\tau, 0)\right) u(0)=0 .
\end{aligned}
$$

Setting $u^{i n t}(\tau, 0)+U(\tau, z)=g(V), u=d g(V) v$ (we omit the dependence with respect to $\tau$ in this section since $\tau$ is fixed), we rewrite the problem in the primitive variable. Hence we have to study the equation

$$
\begin{aligned}
& \lambda A^{0} v+A v^{\prime}-\left(b v^{\prime}\right)^{\prime}=A^{\prime} v+(C v)^{\prime}, \\
& L v(0)=\left(\begin{array}{c}
l w(0) \\
z(0)
\end{array}\right)=0
\end{aligned}
$$


where $v=\left(\begin{array}{c}w \\ z\end{array}\right), A^{0}(z)=d g(V), A(z)=d f(V), b(z)=b(V)$, and $C h=$ $d b(V) h V^{\prime}$. Note that we have the estimates

$$
\left|A^{0^{\prime}}\right|+\left|A^{\prime}\right|+|C|+\left|C^{\prime}\right|+\left|S^{\prime}\right| \leq M\left|V^{\prime}\right|
$$

for some $M>0$, where $S(z)$ stands for $S(V)$.

Moreover, note that thanks to (H1),

$$
\Pi_{w}(C v)^{\prime}=0
$$

where $\Pi_{w}\left(\begin{array}{l}w \\ z\end{array}\right)=w$.

Let us assume that there exists a nonzero solution of (18), (19); without loss of generality, we assume that

$$
\|v\|=1 \text {. }
$$

In this section, since we deal with functions that take complex values, we denote by $u \cdot v$ the scalar product of $\mathbb{C}^{n}$,

$$
u \cdot v=\sum_{i=1}^{n} u_{i} \bar{v}_{i}
$$

and by $|\cdot|^{2}$ the associated norm. We then define

$$
\|v\|^{2}=\int_{0}^{+\infty} v \cdot v d x, \quad(u, v)=\int_{0}^{+\infty} u(x) \cdot v(x) d x
$$

We split the proof of the theorem into several lemmas. We will collect all the estimates at the end of the section to reach our conclusion.

We first give an energy estimate in the same spirit as in the totally parabolic case [2], 4] or in the pure Dirichlet's boundary condition case [12]:

Lemma 4. Assume that $v$ is a solution of (18), (19) that satisfies (22). Then, when $\delta$ is sufficiently small, we have the estimate

$$
\begin{gathered}
\mathcal{R} e \lambda+\beta\left\|z^{\prime}||^{2}+\alpha|w(0)|^{2} \leq C \int_{0}^{+\infty}\left|V^{\prime} \| w\right|^{2},\right. \\
\mathcal{R} e \lambda+\beta \| z^{\prime}||^{2}+\alpha|w(0)|^{2} \leq C \delta\left(|w(0)|^{2}+\left\|v^{\prime}\right\|^{2}\right), \\
|\mathcal{I} m \lambda| \leq C\left(\delta+\left\|v^{\prime}\right\|\right) .
\end{gathered}
$$

Note that the first estimate (23) gives

$$
\mathcal{R} e \lambda \leq C \delta
$$

Proof. We first use the same energy estimate as in the strictly parabolic case [2], [4] and the full Dirichlet case [12]. We take the Hermitian product of (16) by $S v$ (in this section, we will denote $S(V)$ by $S$ for the sake of simplicity) and we take the real part, getting

$$
\mathcal{R} e \lambda\left(S A^{0} v, v\right)+\mathcal{R} e\left(S A v^{\prime}, v\right)-\mathcal{R} e\left(S\left(b v^{\prime}\right)^{\prime}, v\right)=\mathcal{R} e\left(S A^{\prime} v, v\right)+\mathcal{R} e\left(S(C v)^{\prime}, v\right) .
$$


Since $S A$ is symmetric, we get

$$
\begin{aligned}
\mathcal{R} e\left(S A v^{\prime}, v\right) & =-\frac{1}{2}\left((S A)^{\prime} v, v\right)-\frac{1}{2} S A v(0) \cdot v(0) \\
& \geq-\frac{1}{2}\left((S A)^{\prime} v, v\right)+\alpha|w(0)|^{2}
\end{aligned}
$$

thanks to (H6). Note that

$$
\left.\mid(S A)^{\prime} v, v\right)\left.\left|\leq C \int_{0}^{+\infty}\right| V^{\prime}|| v\right|^{2} d x
$$

Next, integrating by parts, we have

$$
\mathcal{R} e\left(S\left(b v^{\prime}\right)^{\prime}, v\right)=-\mathcal{R} e\left(S b v^{\prime}, v^{\prime}\right)-\mathcal{R} e\left(\left(S^{\prime} b v^{\prime}, v\right)-\mathcal{R} e S b v^{\prime}(0) \cdot v(0) .\right.
$$

Thanks to $\left(\mathrm{H}^{\prime}\right)$, we have

$$
\mathcal{R} e\left(S b v^{\prime}, v^{\prime}\right) \geq \beta\left|z^{\prime}\right|^{2}
$$

moreover, we have

$$
\mathcal{R} e S b v^{\prime}(0) \cdot v(0)=0
$$

thanks to the structure of the matrix $b$ given by (H1) and (19). Using again (H1) and (44), we have

$$
\left(\left(S^{\prime} b v^{\prime}, v\right)\right)=\left(S_{z}^{\prime} b_{1} z^{\prime}, z\right) \leq C\left(\eta\left\|z^{\prime}\right\|^{2}+\frac{1}{\eta} \int_{0}^{+\infty}\left|V^{\prime} \| z\right|^{2}\right)
$$

for every $\eta>0$ by using the Young inequality. Moreover, we have

$$
\left|\left(A^{\prime} v, v\right)\right| \leq C \int_{0}^{+\infty}\left|V^{\prime}\right||v|^{2} d z
$$

and

$$
\begin{aligned}
\left|\left(S(C v)^{\prime}, v\right)\right| & =\left|\left(S C v, v^{\prime}\right)+\left(S^{\prime} C v, v\right)\right| \\
& \leq C \int_{0}^{+\infty}\left|V^{\prime}\right|\left|v \| z^{\prime}\right| \leq C\left(\eta\left\|z^{\prime}\right\|^{2}+\frac{1}{\eta} \int_{0}^{+\infty}\left|V^{\prime}\right||v|^{2}\right)
\end{aligned}
$$

thanks to (H1) and (20).

Collecting these various inequalities, we have shown

$\mathcal{R} e \lambda\|v\|^{2}+\beta\left\|z^{\prime}\right\|^{2}+\alpha|w(0)|^{2} \leq C \eta\left\|z^{\prime}\right\|^{2}+C(\eta) \int_{0}^{+\infty}\left|V^{\prime}\left\|\left.z\right|^{2}+C(\eta) \int_{0}^{+\infty}\left|V^{\prime} \| v\right|^{2}\right.\right.$.

To conclude, we first choose $\eta=\frac{\beta}{2}$, then we use $z(0)=0$ through the inequality $|z(x)|^{2} \leq x\left\|z^{\prime}\right\|^{2}$ to get

$$
\int_{0}^{+\infty}\left|V^{\prime}\right||z|^{2} \leq C \int_{0}^{+\infty} x\left|V^{\prime}\left\|\mid z^{\prime}\right\|^{2} \leq C \delta\left\|z^{\prime}\right\|^{2}\right.
$$

and finally, we absorb the terms $C \eta\left\|z^{\prime}\right\|^{2}$ and $C(\eta) \int_{0}^{+\infty}\left|V^{\prime} \| z\right|^{2}$ in the viscous part $\beta\left\|z^{\prime}\right\|^{2}$ if $\delta$ is sufficiently small. This proves (23).

To get (24), we use

$\int_{0}^{+\infty}\left|V^{\prime}\right||w|^{2} \leq C\left(\int_{0}^{+\infty} x\left|V^{\prime}\right||| w^{\prime} \|^{2}+|w(0)|^{2} \int_{0}^{+\infty}\left|V^{\prime}\right|\right) \leq C \delta\left(|w(0)|^{2}+\| w^{\prime}||^{2}\right)$. 
To prove (25), we also take the scalar product of (16) by $S v$, we take the imaginary part and we only use

$$
\operatorname{Im}\left(S b v^{\prime \prime}, v\right)=-\mathcal{I} m\left(S b v^{\prime}, v^{\prime}\right)-\mathcal{I} m\left((S b)^{\prime} v^{\prime}, v\right) \leq C\left(\left\|z^{\prime}\right\|^{2}+\left\|v^{\prime}\right\|\|v\|\right) .
$$

We get

$$
\mathcal{I} m \lambda\|v\|^{2} \leq C\left(\left\|z^{\prime}\right\|^{2}+\left\|v^{\prime}\right\|\|v\|+\delta\|v\|^{2}\right) .
$$

To conclude, it suffices to use (23), which gives, in particular,

$$
\left\|z^{\prime}\right\|^{2} \leq C \delta\|w\|^{2}
$$

and the normalization assumption (22).

In the case of a pure Dirichlet boundary condition, a weighted energy estimate on the hyperbolic part of the system (that is to say on the first $n-r$ equations) was used in [12] to bound the term

$$
\int_{0}^{+\infty}\left|V^{\prime}\right||w|^{2}
$$

This estimate was similar to the one used by Goodman [3] for the stability of viscous shock profiles. This was efficient because of the upwind propagation. In our more general setting, we use an energy estimate of "Kawashima's type" [6], 9].

Lemma 5. Assume that $v$ is a solution of (18), (19) that satisfies (22). Then for sufficiently small $\delta$, we have

$$
\left\|v^{\prime}\right\|^{2} \leq C\left(\left\|z^{\prime}\right\|^{2}+\left\|z^{\prime \prime}\right\|^{2}+(\mathcal{R} e \lambda)^{2}+\delta|w(0)|^{2}\right) .
$$

Proof. We use the matrix $k$ given by (10). We apply $k$ to (16), we take the scalar product by $v^{\prime}$ and we take the real part. Using $\mathcal{R} e\left(k A v^{\prime}, v^{\prime}\right)=\left((k A)^{s} v^{\prime}, v^{\prime}\right)$, we get

$$
\begin{aligned}
\mathcal{R} e\left(\lambda\left(k A^{0} v, v^{\prime}\right)\right)+\left((k A)^{s} v^{\prime}, v^{\prime}\right) & \leq C\left(\left\|z ^ { \prime \prime } \left|\left\||| z^{\prime}\right\|+\delta\left\|v^{\prime}||\right\| z^{\prime}\left\|+\int_{0}^{+\infty}\left|V^{\prime} \| z\right|^{2}\right)\right.\right.\right. \\
& \leq C\left(\left\|\left.z^{\prime \prime}\left|\left\||| z^{\prime}\right\|+\delta\left\|v^{\prime}\right\|^{2}+\delta\right| w(0)\right|^{2}\right) .\right.
\end{aligned}
$$

Here we have used the estimates (28) and (29).

Using that $k A^{0}$ is skew-Hermitian, we have $\left(k A^{0} v, v^{\prime}\right) \in \mathbb{R}$ since

$$
\left(k A^{0} v, v^{\prime}\right)=k A^{0} v(0) \cdot v(0)-\left(\left(k A^{0}\right)^{\prime} v, v\right)-\left(k A^{0} v^{\prime}, v\right)=-\left(k A^{0} v^{\prime}, v\right) .
$$

Consequently, we have

$$
\left|\mathcal{R} e\left(\lambda\left(k A^{0} v, v^{\prime}\right)\right)\right|=\left|\mathcal{R} e(\lambda)\left(k A^{0} v, v^{\prime}\right)\right| \leq C \mathcal{R} e \lambda\|v\|\left\|v^{\prime}\right\| .
$$

Since we have the estimate (10)

$$
\left((k A)^{s} v^{\prime}, v^{\prime}\right) \geq \theta\left\|v^{\prime}||^{2}-C\right\| z^{\prime} \|^{2},
$$

we get

$$
\left\|v^{\prime}\right\|^{2} \leq C\left(\left\|z^{\prime}\right\|^{2}+\left\|z^{\prime \prime}|||| v^{\prime}||+\mathcal{R} e \lambda\right\| v\left\||| v^{\prime}\right\|+\delta|| v^{\prime} \|^{2}+\delta|w(0)|^{2}\right),
$$

and hence choosing $\eta>0$ sufficiently small, using the Young inequality and (9), we have

$$
\left\|v^{\prime}\right\|^{2} \leq C(\eta)\left(\left\|z^{\prime}\right\|^{2}+\frac{(\mathcal{R} e \lambda)^{2}}{\eta}\|v\|^{2}+\frac{1}{\eta}\left\|z^{\prime \prime}\right\|^{2}+\delta|w(0)|^{2}\right) .
$$

Consequently (30) is proved. 
To end the proof of the theorem we would want to estimate $\left\|z^{\prime \prime}\right\|$ with respect to $\left\|v^{\prime}\right\|$ and $\|v\|$.

Lemma 6. Assume that $v$ is a solution of (18), (19) that satisfies (22). Then, when $\delta$ is sufficiently small, we have

$$
\| z^{\prime \prime}||^{2} \leq C\left(\delta|| v^{\prime}||^{2}+\delta|w(0)|^{2}+\left|z^{\prime \prime}(0)\right|^{2}+\left|w^{\prime}(0)\right|^{2}+\left|z^{\prime}(0)\right|^{2}\right) .
$$

Proof. We take the derivative of (16), getting the equation

$$
\lambda A^{0} v^{\prime}+A v^{\prime \prime}-\left(b v^{\prime \prime}\right)^{\prime}=O\left(\left|V^{\prime}\right|\right)\left(|v|+\left|z^{\prime \prime}\right|+\left|v^{\prime}\right|\right)+(C v)^{\prime \prime} .
$$

The proof is very similar to the proof of (23), in that we take the scalar product of the equation by $S v^{\prime}$ and we do an integration by parts. The "boundary" terms do not vanish since $v^{\prime}(0)$ does not satisfy the boundary condition (19). We just point out that to bound the term $\left(\left(S(C v)^{\prime \prime}, v^{\prime}\right)\right.$ we also do an integration by parts as in (27) to get an estimate independent of $\left\|v^{\prime \prime}\right\|$.

2.1. Proof of Theorem 3. We now give the proof of Theorem 3. To conclude, we first have to eliminate $z^{\prime \prime}(0)$ and $v^{\prime}(0)$ in (32).

We first express $w^{\prime}(0)$, thanks to the hyperbolic part of equation (18):

$$
\lambda w+A_{1} w^{\prime}+A_{2} z^{\prime}=O\left(\left|V^{\prime}\right|\right)\left(|v|+\left|z^{\prime}\right|\right),
$$

where $A=\left(\begin{array}{cc}A_{1} & A_{2} \\ A_{2} & A_{4}\end{array}\right)$. Note that we make a crucial use of (21).

Since the boundary is noncharacteristic for the viscous system, $A_{1}$ is nonsingular; moreover, thanks to (23), 25), we have

$$
|\lambda|^{2} \leq \delta+\left\|v^{\prime}\right\|^{2}
$$

We deduce

$$
\begin{aligned}
\left|w^{\prime}(0)\right|^{2} & \leq C\left(\delta|w(0)|^{2}+\left|z^{\prime}(0)\right|^{2}+|w(0)|^{2}\left\|v^{\prime}\right\|^{2}\right) \\
& \leq C\left(\delta|w(0)|^{2}+\left|z^{\prime}(0)\right|^{2}+\delta|| v^{\prime}||^{2}\right)
\end{aligned}
$$

since thanks to (23), we have $|w(0)|^{2} \leq C \delta$.

The next step is to estimate $\left|z^{\prime}(0)\right|$. We use the classical Sobolev inequality

$$
\left|z^{\prime}(0)\right|^{2} \leq 2\left\|z^{\prime \prime} \mid\right\|\|z\| \leq \eta\left\|z^{\prime \prime}\right\|^{2}+\frac{1}{\eta}\|z\|^{2}
$$

for every $\eta>0$. Hence it suffices to estimate $z^{\prime \prime}(0)$ in (32). We use the parabolic part of the equation

$$
\lambda \tilde{g}(V) z+A_{3} w^{\prime}+A_{4} z^{\prime}-b_{1} z^{\prime \prime}=O\left(\left|V^{\prime}\right|\right)\left(|v|+\left|v^{\prime}\right|\right)+O(|\lambda||w|) .
$$

We get, thanks to (23), (25), (34), and (35),

$$
\left|z^{\prime \prime}(0)\right|^{2} \leq C\left(\delta|w(0)|^{2}+\left(\delta+\frac{1}{\eta}\right)\left\|z^{\prime}\right\|^{2}+\delta\left\|v^{\prime}\right\|^{2}+\eta \| z^{\prime \prime}||^{2}\right) .
$$

Next, we choose $\eta$ such that $C \eta<1$, and we replace (34), (35), (36) in (32), getting

$$
\left\|z^{\prime \prime}\right\|^{2} \leq C\left(\delta|| v^{\prime}\left\|^{2}+\right\| z^{\prime} \|^{2}+\delta|w(0)|^{2}\right) .
$$

Finally, collecting (23), (30) and (37), we have shown that

$$
(\mathcal{R} e \lambda)(1-C \delta)+(\beta-C \delta)\left\|z^{\prime}\right\|^{2}+(\alpha-C \delta)|w(0)|^{2} \leq 0 .
$$


Hence if $\delta$ is sufficiently small, this gives if $\mathcal{R} e \lambda \geq 0, z=0$ and $w(0)=0$. The hyperbolic part of the equation then becomes a first-order ordinary differential equation involving only $w$ :

$$
w^{\prime}=\left(A_{1}\right)^{-1}\left(-\lambda w+O\left(\left|V^{\prime}\right|\right) w\right)
$$

with the boundary condition $w(0)=0$. Consequently we also get $w=0$. This ends the proof of Theorem 3 .

\section{NONLinear Stability}

In this section we prove Theorem 1. We use the form (3) of the system. Setting $u^{\varepsilon}=g\left(v^{\varepsilon}\right)$ and $u^{a p p}=g\left(v^{a p p}\right)$, we have the two systems

$$
\begin{aligned}
& g\left(v^{\varepsilon}\right)_{t}+\left(f\left(v^{\varepsilon}\right)_{x}=\varepsilon\left(b\left(v^{\varepsilon}\right) v_{x}^{\varepsilon}\right)_{x},\right. \\
& L v^{\varepsilon}(t, 0)=g, \\
& v^{\varepsilon}(0, x)=v_{0}(x)
\end{aligned}
$$

and

$$
\begin{aligned}
& g\left(v^{a p p}\right)_{t}+\left(f\left(v^{a p p}\right)_{x}=\varepsilon\left(b\left(v^{a p p}\right) v_{x}^{a p p}\right)_{x}+R^{\varepsilon},\right. \\
& L v^{a p p}(t, 0)=g, \\
& v^{a p p}(0, x)=v_{0}(x) .
\end{aligned}
$$

Setting $v^{\varepsilon}=v^{a p p}+v$ (we omit the dependence of $v$ in $\varepsilon$ ), we rewrite our problem as

$$
A^{0} \partial_{t} v+A \partial_{x} v-\varepsilon \partial_{x}\left(b \partial_{x} v\right)=R^{\varepsilon}+M^{0}+M^{1}+M^{2},
$$

where

$$
\begin{aligned}
A^{0} & =d g\left(v^{a p p}+v\right), \\
A & =A^{1}=d f\left(v^{a p p}+v\right), \\
b & =b\left(v^{a p p}+v\right), \\
M^{0} & =\left(d g\left(v^{a p p}+v\right)-d g\left(v^{a p p}\right)\right) \partial_{t} v^{a p p}, \\
M^{1} & =\left(d f\left(v^{a p p}+v\right)-d f\left(v^{a p p}\right)\right) \partial_{x} v^{a p p}, \\
M^{2} & =\varepsilon \partial_{x}\left(\left(b\left(v^{a p p}+v\right)-b\left(v^{a p p}\right)\right) \partial_{x} v^{a p p}\right) .
\end{aligned}
$$

Note that $v$ satisfies the boundary condition

$$
L v(t, 0)=0
$$

and the initial condition

$$
v(0, x)=0 .
$$

We choose $C$ sufficiently large such that

$$
Q^{\varepsilon} \leq C \varepsilon^{N},
$$

where

$$
\begin{aligned}
Q^{\varepsilon}= & \left\|R^{\varepsilon}\right\|^{2}+\varepsilon^{2}\left\|\partial_{t} R^{\varepsilon}\right\|^{2}+\varepsilon^{2}\left\|\partial_{x} R^{\varepsilon}\right\|^{2}+\varepsilon^{4}\left\|\partial_{t t} R^{\varepsilon}\right\|^{2} \\
& +\varepsilon^{4}\left\|\partial_{t x} R^{\varepsilon}\right\|^{2}+\left|R^{\varepsilon}(t, 0)\right|^{2}+\varepsilon^{2}\left|\partial_{t} R^{\varepsilon}(t, 0)\right|^{2}
\end{aligned}
$$


for some large $N$ which will be chosen later.

To prove Theorem 1, we use the classical continuous induction argument ([3], [7], [5], [9]). Let us define

$$
\begin{aligned}
E(t)= & \|v(t)\|^{2}+\varepsilon^{2}\left\|\partial_{t} v(t)\right\|^{2}+\varepsilon^{2}\left\|\partial_{x} v(t)\right\|^{2}+\varepsilon^{4}\left\|\partial_{t t} v\right\|^{2}+\varepsilon^{4}\left\|\partial_{t x} v(t)\right\|^{2} \\
& +\int_{0}^{t} \varepsilon\left\|\partial_{x} v(s)\right\|^{2}+\varepsilon^{3}\left\|\partial_{t x} v(s)\right\|^{2}+\varepsilon^{3}\left\|\partial_{x x} z\right\|^{2} d s \\
& +\int_{0}^{t} \varepsilon^{5}\left\|\partial_{x x t} z(s)\right\|^{2}+\varepsilon^{5}\left\|\partial_{t t x} z(s)\right\|^{2} d s \\
& +\int_{0}^{t}|w(s, 0)|^{2}+\varepsilon^{2}\left|\partial_{t} w(s, 0)\right|^{2}+\varepsilon^{4}\left|\partial_{t t} w(t, 0)\right|^{2} d s .
\end{aligned}
$$

Note that thanks to (39), (40), (41), we have

$$
E(0) \leq C \varepsilon^{N} .
$$

Using the classical short-time theory, we define

$$
\begin{gathered}
T^{*}=\sup \left\{T ^ { \varepsilon } \in [ 0 , T ] , \exists \text { a solution of (138), (39), (40) on } \left[0, T^{\varepsilon}[\right.\right. \\
\text { such that } \left.\forall t \in\left[0, T^{\varepsilon}\right), E(t) \leq \varepsilon^{N_{1}}\right\}
\end{gathered}
$$

where we choose $N_{1}<N$. There are two possibilities:

(1) $T^{*}=T$,

(2) $T^{*}<T$, and $E\left(T^{*}\right)=\varepsilon^{N_{1}}$.

In the following, we show by an energy estimate that we cannot be in the second case. This will show Theorem 1.

Let us define $a\left(\frac{x}{\varepsilon}\right)$ as

$$
a\left(\frac{x}{\varepsilon}\right)=\sup _{t \in[0, T]} \sup _{2 \geq \alpha \geq 1,2 \geq \beta \geq 0}\left|\partial_{z}^{\alpha} \partial_{t}^{\beta} V\left(t, \frac{x}{\varepsilon}\right)\right| .
$$

At first we need an elementary lemma about the estimates of the nonlinear quantities that arise in (38).

Lemma 7. $\forall i=0,1$,

$$
\begin{gathered}
\left|\partial_{t} A^{i}\right| \leq C\left(\left|\partial_{t} v\right|_{\infty}\right), \quad\left|\partial_{t}^{2} A^{i}\right| \leq C\left(\left|\partial_{t} v\right|_{\infty}\right)\left(1+\left|\partial_{t t} v\right|\right) \\
\left|\partial_{x} A^{i}\right| \leq C\left(\left|\partial_{x} v\right|_{\infty}\right)\left(1+\frac{1}{\varepsilon} a\left(\frac{x}{\varepsilon}\right)\right) \\
\left|\partial_{t x} A^{i}\right| \leq C\left(\left|\partial_{t} v\right|_{\infty},\left|\partial_{x} v\right|_{\infty}\right)\left(1+\frac{1}{\varepsilon} a\left(\frac{x}{\varepsilon}\right)+\left|\partial_{t x} v\right|\right) .
\end{gathered}
$$

Similar estimates hold for $A^{i}$ replaced by $b, S\left(v^{a p p}+v\right)$ or $k\left(v^{a p p}+v\right)$. Moreover $\forall \alpha \leq 2, \beta \leq 1$,

$$
\begin{aligned}
\left|\partial_{t}^{\alpha} \partial_{x}^{\beta} M^{0}\right| & \leq C\left(|v|_{\infty},\left|\partial_{x} v\right|_{\infty},\left|\partial_{t} v\right|_{\infty}\right) \sum_{\gamma \leq \alpha, \delta \leq \beta}\left(1+\frac{1}{\varepsilon^{\beta-\delta}} a\left(\frac{x}{\varepsilon}\right)\right)\left|\partial_{t}^{\alpha} \partial_{x}^{\delta} v\right|, \\
\left|\partial_{t}^{\alpha} \partial_{x}^{\beta} M^{1}\right| & \leq C\left(|v|_{\infty},\left|\partial_{x} v\right|_{\infty},\left|\partial_{t} v\right|_{\infty}\right) \sum_{\gamma \leq \alpha, \delta \leq \beta}\left(1+\frac{1}{\varepsilon^{\beta-\delta+1}} a\left(\frac{x}{\varepsilon}\right)\right)\left|\partial_{t}^{\alpha} \partial_{x}^{\delta} v\right|, \\
\left|\partial_{t}^{\alpha} M^{2}\right| & \leq C\left(|v|_{\infty},\left|\partial_{x} v\right|_{\infty},\left|\partial_{t} v\right|_{\infty}\right) \sum_{\gamma \leq \alpha, \delta \leq 1}\left(\varepsilon+\frac{1}{\varepsilon^{1-\delta}} a\left(\frac{x}{\varepsilon}\right)\right)\left|\partial_{t}^{\alpha} \partial_{x}^{\delta} v\right| .
\end{aligned}
$$


Remark 8. We point out that $\Pi_{w} \partial_{t}^{\alpha} \partial_{x}^{\beta} M^{2}=0$ where $\Pi_{w}\left(\begin{array}{c}w \\ z\end{array}\right)=w$.

We also point out that, actually, we will not use the case $\alpha=2, \beta=1$ in Lemma 7]

We now come to the proof of our main theorem 1. In the proof, $C$ stands for a number that is independent of $\varepsilon$ but may depend on $T$.

Since by classical Sobolev embeddings, we have

$$
\begin{gathered}
|v|_{\infty}^{2} \leq C \sup _{t \in\left[0, T^{*}\right]}\|v(t)\|_{H^{1}}^{2},\left|\partial_{t} v\right|_{\infty}^{2} \leq C \sup _{t \in\left[0, T^{*}\right]}\left\|\partial_{t} v(t)\right\|_{H^{1}}^{2}, \\
\left|\partial_{x} z\right|_{\infty}^{2} \leq 2 \int_{0}^{T^{*}}\left|\partial_{t x} z(s)\right|_{\infty}\left|\partial_{x} z(s)\right|_{\infty} \leq C\left(\int_{0}^{T *}\left\|\partial_{t x} z(s)\right\|_{H^{1}}^{2}+\left\|\partial_{x} z(s)\right\|_{H^{1}}^{2} d s\right)
\end{gathered}
$$

and since by using the hyperbolic part of equation (38) (that is to say, the $w$ component), the noncharacteristic assumption and Remark 8 , we have

$$
\left|\partial_{x} w\right|_{\infty} \leq C\left(\left|\partial_{t} w\right|_{\infty}+\frac{1}{\varepsilon}|v|_{\infty}+\left|\partial_{x} z\right|_{\infty}\right),
$$

we get the estimate

$$
\left|\partial_{t} v\right|_{\infty}^{2}+\left|\partial_{x} v\right|_{\infty}^{2}+|v|_{\infty}^{2} \leq \frac{C}{\varepsilon^{5}} E\left(T^{*}\right) \leq C \varepsilon^{N_{1}-5}
$$

Consequently, we choose $N \geq 6$ and $5<N_{1}<N$. This allows us to use Lemma 7 Moreover, thanks to (43), we obtain by continuity from (H6) that

$$
S_{w}\left(v^{a p p}+v\right) h\left(v^{a p p}+v\right) X_{1} \cdot X_{1} \leq-\beta\left|X_{1}\right|^{2}, \forall X=\left(\begin{array}{c}
X_{1} \\
X_{2}
\end{array}\right), L X=0
$$

since $v^{a p p}$ satisfies $L v^{a p p}=g$.

Note that our smallness assumption (9) and (6) imply that

$$
\sup _{z \in \mathbb{R}_{+}} a(z)+\int_{0}^{+\infty} a(z) d z+\int_{0}^{+\infty} z a(z) d z \leq C \delta .
$$

As for the spectral stability, there are four steps in the proof. We first make the energy estimate of the totally parabolic case; next we make an estimate of Kawashima's type and an estimate on the space derivative of equation (38). The final step is to estimate the boundary values. For this, we replace (25) in the time evolutionary setting by an energy estimate on the time derivative of the equation.

At first, let us make the same energy estimate as for the totally parabolic case [2], 4]. Using Lemma 4 and

$$
\frac{1}{\varepsilon} \int_{0}^{+\infty} a\left(\frac{x}{\varepsilon}\right)|v|^{2} \leq \delta \varepsilon|| \partial_{x} v \|^{2}+\delta \varepsilon|w(t, 0)|^{2},
$$

$$
\begin{aligned}
\int_{0}^{+\infty} a\left(\frac{x}{\varepsilon}\right)\left|v \| \partial_{x} v\right| & \leq C|| \partial_{x} v\left\|\left(\int_{0}^{+\infty} a\left(\frac{x}{\varepsilon}\right)|v|^{2}\right)^{\frac{1}{2}} \leq C|| \partial_{x} v\right\|\left(\sqrt{\varepsilon} \delta|w(t, 0)|+\varepsilon \delta\left\|\partial_{x} v\right\|\right) \\
& \leq C\left(\varepsilon \delta\left\|\partial_{x} v\right\|^{2}+\delta|w(t, 0)|^{2}\right)
\end{aligned}
$$

we easily get, after absorbing the terms $C \delta|w(t, 0)|^{2}$ by the term $\alpha|w(t, 0)|^{2}$ on the left-hand side,

$$
\partial_{t}\left(S A^{0} v, v\right)+\alpha|w(t, 0)|^{2}+\beta \varepsilon\left\|\partial_{x} z\right\|^{2} \leq C\left(Q^{\varepsilon}+\|v\|^{2}+\varepsilon \delta\left\|\partial_{x} v\right\|^{2}\right),
$$

where $S$ stands for $S\left(u^{a p p}+v\right)$. 
Note that an estimate such as (47) is needed to bound the terms

$$
\left(\varepsilon S \partial_{x}\left(b \partial_{x} v\right), v\right),\left(M_{2}, v\right) \text {. }
$$

Next we replace the estimate (25) of the spectral stability by an estimate on the time derivative $\partial_{t} v$. Since $\partial_{t} v$ still satisfies the boundary condition $L \partial_{t} v=0$, we can perform the same computation as previously on the time derivative of (38). Thanks to Lemma 7, we get

$$
\begin{aligned}
& \partial_{t}\left(S A^{0} \partial_{t} v, \partial_{t} v\right)+\alpha\left|\partial_{t} w(t, 0)\right|^{2}+\beta \varepsilon\left\|\partial_{t x} z\right\|^{2} \\
& \leq C\left(\frac{1}{\varepsilon^{2}} Q^{\varepsilon}+\|v\|^{2}+\left\|\partial_{t} v\right\|^{2}+\left\|\partial_{x} v\right\|^{2}+\frac{1}{\varepsilon} \int_{0}^{+\infty} a\left(\frac{x}{\varepsilon}\right)\left|\partial_{t} v\right|^{2} d x\right) .
\end{aligned}
$$

We do not give more details since all the ideas of the computation have been used. We just point out that we have used that

$$
\begin{aligned}
\frac{1}{\varepsilon} \int_{0}^{+\infty} a\left(\frac{x}{\varepsilon}\right)\left|v \| \partial_{t} v\right| d x & \leq \frac{C}{\varepsilon}\left\|\partial_{t} v\right\|\left(\int_{0}^{+\infty} a\left(\frac{x}{\varepsilon}\right)|v|^{2} d x\right)^{\frac{1}{2}} \\
& \leq \frac{C}{\varepsilon}\left\|\partial_{t} v\right\|\left(\delta \sqrt{\varepsilon}|w(t, 0)|+\delta \varepsilon\left\|\partial_{x} v\right\|\right) \\
& \leq C\left(\delta\left\|\partial_{t} v\right\|^{2}+\delta\left\|\partial_{x} v\right\|^{2}+\frac{\delta}{\varepsilon}|w(t, 0)|^{2}\right)
\end{aligned}
$$

and that to bound the term

$$
\varepsilon\left(S \partial_{x}\left(\partial_{t} b \partial_{x} v\right), \partial_{t} v\right)
$$

we perform an integration by parts and use the block assumption (H1) and (44). This term is then dominated by

$$
C\left(\left\|\partial_{t} z\right\|^{2}+\frac{\delta}{\varepsilon \eta}\left\|\partial_{x} z\right\|^{2}+\varepsilon \eta\left\|\partial_{t x} z\right\|^{2}\right)
$$

for every $\eta>0$. We absorb the last factor by the term $\beta \varepsilon\left\|\partial_{t x} z\right\|^{2}$ in the left-hand side by choosing $\eta$ sufficiently small.

Note that for the moment, we do not use an inequality similar to (46) to bound terms such as

$$
\int_{0}^{+\infty} a\left(\frac{x}{\varepsilon}\right)\left|\partial_{t} v\right|^{2} d x
$$

We bound this term by expressing $\partial_{t} v$, thanks to equation (38) and by using estimates such as (46) and (47). Then we get

$$
\frac{1}{\varepsilon} \int_{0}^{+\infty} a\left(\frac{x}{\varepsilon}\right)\left|\partial_{t} v\right|^{2} d x \leq C\left(\frac{\delta}{\varepsilon}\left\|\partial_{x} v\right\|^{2}+\delta \varepsilon\left\|\partial_{x x} z\right\|^{2}+\frac{\delta}{\varepsilon^{2}}|w(t, 0)|^{2}\right) .
$$

Note that the factor $\frac{\delta}{\varepsilon^{2}}|w(t, 0)|^{2}$ comes from

$$
\frac{1}{\varepsilon} \int_{0}^{+\infty} a\left(\frac{x}{\varepsilon}\right)\left|M_{1}\right|^{2} d x \leq \frac{1}{\varepsilon} \int_{0}^{+\infty} a\left(\frac{x}{\varepsilon}\right)\left(1+\frac{\delta}{\varepsilon^{2}}\right)|v|^{2} d x .
$$

The estimate (46) gives

$$
\frac{1}{\varepsilon^{3}} \int_{0}^{+\infty} a\left(\frac{x}{\varepsilon}\right)|v|^{2} \leq C\left(\frac{\delta}{\varepsilon^{2}}|w(t, 0)|^{2}+\frac{\delta}{\varepsilon}\left\|\partial_{x} v\right\|^{2}\right) .
$$


Replacing (51) in (49) yields

$$
\begin{aligned}
& \partial_{t}\left(S A^{0} \partial_{t} v, \partial_{t} v\right)+\alpha\left|\partial_{t} w(t, 0)\right|^{2}+\beta \varepsilon\left\|\partial_{t x} z\right\|^{2} \\
& \leq C\left(\frac{1}{\varepsilon^{2}} Q^{\varepsilon}+\|v\|^{2}+\left\|\partial_{t} v\right\|^{2}+\left(1+\frac{\delta}{\varepsilon}\right)\left\|\partial_{x} v\right\|^{2}+\delta \varepsilon\left\|\partial_{x x} z\right\|^{2}+\frac{\delta}{\varepsilon^{2}}|w(t, 0)|^{2}\right) .
\end{aligned}
$$

As for the spectral stability, the next step is to use the "Kawashima" estimate [6], 9]. We apply $k\left(v^{a p p}+v\right)$ to (38) and we take the scalar product by $\partial_{x} v$ :

$$
\begin{aligned}
& \left(k A^{0} \partial_{t} v, \partial_{x} v\right)+\left((k A)^{s} \partial_{x} v, \partial_{x} v\right)-\varepsilon\left(k \partial_{x}\left(b \partial_{x} v\right) \partial_{x} v\right) \\
& =\left(k R^{\varepsilon}+k M^{0}+k M^{1}+k M^{2}, \partial_{x} v\right) .
\end{aligned}
$$

We use the crucial estimate (10). This gives

$$
\left((k A)^{s} \partial_{x} v, \partial_{x} v\right) \geq \theta\left\|\partial_{x} v\right\|^{2}-C\left\|\partial_{x} z\right\|^{2} .
$$

Next we write for every $\eta>0$,

$$
\begin{aligned}
\varepsilon\left|\left(k \partial_{x}\left(b \partial_{x} v\right), \partial_{x} v\right)\right| & =\left|\varepsilon\left(k \partial_{x} b \partial_{x} v, \partial_{x} v\right)+\varepsilon\left(k b \partial_{x x} v \partial_{x} v\right)\right| \\
& \leq C\left((\varepsilon+\delta)\left\|\partial_{x} v\right\|^{2}+\varepsilon\left\|\partial_{x x} z\right\| \partial_{x} v \|\right) \\
& \leq C\left((\varepsilon+\delta+\eta)\left\|\partial_{x} v\right\|^{2}+\frac{\varepsilon^{2}}{\eta}\left\|\partial_{x x} z\right\|^{2}\right),
\end{aligned}
$$

where here we have used the block structure assumption (H1) and the Young inequality. Using Lemma 7, the Young inequality, and (47) we have

$$
\begin{aligned}
\left|\left(k M^{1}, \partial_{x} v\right)\right| & \leq C \int_{0}^{+\infty}\left(1+\frac{1}{\varepsilon} a\left(\frac{x}{\varepsilon}\right)\right)|v|\left|\partial_{x} v\right| d x \\
& \leq C\left(\frac{1}{\eta}\|v\|^{2}+(\eta+\delta)\left\|\partial_{x} v\right\|^{2}+\frac{\delta}{\varepsilon}|w(t, 0)|^{2}\right) .
\end{aligned}
$$

By the same method, we get similar estimates for $\left(k M^{0}, \partial_{x} v\right)$ and $\left(k M^{2}, \partial_{x} v\right)$. To handle $\left(k A^{0} \partial_{t} v, \partial_{x} v\right)$, we write

$$
\left(k A^{0} \partial_{t} v, \partial_{x} v\right)=\partial_{t}\left(k A^{0} v, \partial_{x} v\right)-\left(\partial_{t}\left(k A^{0}\right) v, \partial_{x} v\right)-\left(k A^{0} v, \partial_{t x} v\right) .
$$

Performing an integration by parts in the last factor above, we get

$$
\begin{aligned}
\left(k A^{0} \partial_{t} v, \partial_{x} v\right)= & \partial_{t}\left(k A^{0} v, \partial_{x} v\right)-\left(\partial_{t}\left(k A^{0}\right) v, \partial_{x} v\right)+k A^{0}(t, 0) v(t, 0) \cdot \partial_{t} v(t, 0) \\
& +\left(\partial_{x}\left(k A^{0}\right) v, \partial_{t} v\right)+\left(k A^{0} \partial_{x} v, \partial_{t} v\right)
\end{aligned}
$$

and hence, since $k A^{0}$ is skew-symmetric,

$$
\begin{aligned}
\left(k A^{0} \partial_{t} v, \partial_{x} v\right)=\frac{1}{2}( & \partial_{t}\left(k A^{0} v, \partial_{x} v\right)-\left(\partial_{t}\left(k A^{0}\right) v, \partial_{x} v\right) \\
& \left.+k A^{0}(t, 0) v(t, 0) \cdot \partial_{t} v(t, 0)+\left(\partial_{x}\left(k A^{0}\right) v, \partial_{t} v\right)\right) .
\end{aligned}
$$

To bound $\left(\partial_{x}\left(k A^{0}\right) v, \partial_{t} v\right)$, we use

$$
\begin{aligned}
\frac{1}{\varepsilon} \int_{0}^{+\infty} a\left(\frac{x}{\varepsilon}\right)\left|v \| \partial_{t} v\right| d x & \leq C\left(\frac{1}{\varepsilon^{2}} \int_{0}^{+\infty} a\left(\frac{x}{\varepsilon}\right)|v|^{2} d x+\int_{0}^{+\infty} a\left(\frac{x}{\varepsilon}\right)\left|\partial_{t} v\right|^{2} d x\right) \\
& \leq C\left(\delta\left\|\partial_{x} v\right\|^{2}+\delta \varepsilon^{2}\left\|\partial_{x x} z\right\|^{2}+\frac{\delta}{\varepsilon}|w(t, 0)|^{2}\right),
\end{aligned}
$$

thanks to (46) and (51). 
This yields

$$
\begin{aligned}
& \left(k A^{0} \partial_{t} v, \partial_{x} v\right)=\frac{1}{2} \partial_{t}\left(k A^{0} v, \partial_{x} v\right) \\
& +O\left(\frac{1}{\varepsilon}\|v\|^{2}+(\varepsilon+\delta)\left\|\partial_{x} v\right\|^{2}+\frac{\eta+\delta}{\varepsilon}|w(t, 0)|^{2}+\frac{\varepsilon}{\eta}\left|\partial_{t} w(t, 0)\right|^{2}+\delta \varepsilon^{2}\left\|\partial_{x x} z\right\|^{2}\right)
\end{aligned}
$$

for any $\eta>0$ by use of the Young inequality. Consequently, collecting (54), (55), (56), choosing $\eta$ sufficiently small and using our assumption (9) to absorb $(\varepsilon+$ $\delta)\left\|\partial_{x} v\right\|^{2}$ in the left-hand side if $\delta$ is sufficiently small, we get from (53),

$$
\begin{aligned}
\partial_{t}\left(k A^{0} v, \partial_{x} v\right)+\theta\left\|\partial_{x} v\right\|^{2} \leq C & \left(\frac{1}{\varepsilon} Q^{\varepsilon}+\frac{1}{\varepsilon}\|v\|^{2}+\frac{1}{\varepsilon}(\delta+\eta)|w(t, 0)|^{2}\right. \\
& \left.+\frac{\varepsilon}{\eta}\left|\partial_{t} w(t, 0)\right|^{2}+\frac{\varepsilon^{2}}{\eta}\left\|\partial_{x x} z\right\|^{2}\right) .
\end{aligned}
$$

In conclusion, it remains to estimate $\left\|\partial_{x x} z\right\|^{2}$. We take the derivative of (38) with respect to $x$ and we perform an energy estimate similar to (23), but now the boundary terms in the integration by parts do not vanish. Using Lemma 7 and estimates such as (46), (47), and (50), we get

$$
\begin{aligned}
& \partial_{t}\left(S A^{0} \partial_{x} v, \partial_{x} v\right)+\beta \varepsilon\left\|\partial_{x x} z\right\|^{2} \\
& \leq C\left(\frac{Q^{\varepsilon}}{\varepsilon^{2}}+\left(1+\frac{\delta}{\varepsilon}\right)\|v\|^{2}+\left(1+\frac{\delta}{\varepsilon}\right)\left\|\partial_{x} v\right\|^{2}+\frac{\delta}{\varepsilon^{2}}|w(t, 0)|^{2}\right. \\
& \left.\quad+\left|\partial_{x} v(t, 0)\right|^{2}+\varepsilon^{2}\left|\partial_{x x} z(t, 0)\right|^{2}\right) .
\end{aligned}
$$

Note that to estimate the term $\left(\partial_{x} M^{2}, \partial_{x} v\right)$ we have perfomed an integration by parts to avoid that terms involving $\left\|\partial_{x x} v\right\|$ appear and that to estimate $\left(S \partial_{x} A^{0} \partial_{t} v, \partial_{x} v\right)$, we write

$$
\left|\left(S \partial_{x} A^{0} \partial_{t} v, \partial_{x} v\right)\right| \leq C\left(\left(1+\frac{\delta}{\varepsilon}\right)\left\|\partial_{x} v\right\|^{2}+\left\|\partial_{t} v\right\|^{2}+\frac{1}{\varepsilon} \int_{0}^{+\infty} a\left(\frac{x}{\varepsilon}\right)\left|\partial_{t} v\right|^{2} d x\right)
$$

and we use (51).

As for the spectral stability, the next step is to estimate the boundary values $\partial_{x} v(t, 0)$ and $\partial_{x x} z(t, 0)$. We first write the analogue of (35),

$$
\left|\partial_{x} z(t, 0)\right|^{2} \leq C\left(\varepsilon \eta\left\|\partial_{x x} z\right\|^{2}+\frac{1}{\varepsilon \eta} \|\left.\partial_{x} z\right|^{2}\right)
$$

for some $\eta$ sufficiently small so that the term

$$
\varepsilon \eta\left\|\partial_{x x} z\right\|^{2}
$$

will be absorbed by the left-hand side of (158).

To estimate $\partial_{x} w(t, 0)$, we use the hyperbolic part of the equation, the fact that the boundary is noncharacteristic and Lemma 7 , getting the estimate

$$
\begin{aligned}
\left|\partial_{x} w(t, 0)\right|^{2} \leq & C\left(\left|R^{\varepsilon}(t, 0)\right|^{2}+\left|\partial_{t} w(t, 0)\right|^{2}+\left|\partial_{x} z(t, 0)\right|^{2}+\left(1+\frac{\delta}{\varepsilon^{2}}\right)|w(t, 0)|^{2}\right) \\
60) \leq C & \left(\left|R^{\varepsilon}(t, 0)\right|^{2}+\left|\partial_{t} w(t, 0)\right|^{2}+\left(1+\frac{\delta}{\varepsilon^{2}}\right)|w(t, 0)|^{2}\right. \\
& \left.+\varepsilon \eta\left\|\partial_{x x} z\right\|^{2}+\frac{1}{\varepsilon \eta}\left\|\partial_{x} z\right\|^{2}\right) .
\end{aligned}
$$


To estimate $\partial_{x x} z(t, 0)$, we use the parabolic part of the equation. This yields

$$
\begin{aligned}
\varepsilon^{2}\left|\partial_{x x} z(t, 0)\right|^{2} \leq C & \left(\left|R^{\varepsilon}(t, 0)\right|^{2}+\left|\partial_{t} w(t, 0)\right|^{2}+\varepsilon \eta\left\|\partial_{x x} z\right\|^{2}\right. \\
& \left.+\frac{1}{\varepsilon \eta}\left\|\partial_{x} z\right\|^{2}+\left(1+\frac{\delta}{\varepsilon^{2}}\right)|w(t, 0)|^{2}\right) .
\end{aligned}
$$

Next, putting (59), (60), (61) in (58) gives the bound

$$
\begin{aligned}
& \partial_{t}\left(S A^{0} \partial_{x} v, \partial_{x} v\right)+\varepsilon\left\|\partial_{x x} z\right\|^{2} \\
& \leq C\left(\frac{1}{\varepsilon^{2}} Q^{\varepsilon}+\left(1+\frac{\delta}{\varepsilon}\right)\|v\|^{2}+\left(1+\frac{\delta}{\varepsilon}\right)\left\|\partial_{x} v\right\|^{2}+\frac{\delta}{\varepsilon^{2}}|w(t, 0)|^{2}\right) .
\end{aligned}
$$

Finally, we consider (48) $+\varepsilon^{2}(\underline{52})+\varepsilon(157)+\Gamma \varepsilon^{2}(\underline{62})$ with $\Gamma>0$ sufficiently large and independent of $\varepsilon$. Integrating from 0 to $t$, we get the estimate

$$
\begin{aligned}
& \|v(t)\|^{2}+\varepsilon^{2}\left\|\partial_{t} v(t)\right\|^{2}+\varepsilon^{2} \Gamma\left\|\partial_{x} v(t)\right\|^{2} \\
& +\beta\left(\int_{0}^{t} \varepsilon\left\|\partial_{x} v(s)\right\|^{2}+\varepsilon^{3}\left\|\partial_{t x} z(s)\right\|^{2}+\Gamma \varepsilon^{3}\left\|\partial_{x x} z(s)\right\|^{2} d s\right) \\
& +\alpha\left(|w(t, 0)|^{2}+\varepsilon^{2}\left|\partial_{t} w(t, 0)\right|^{2}\right)+\varepsilon\left(k A v, \partial_{x} v\right)(t) \\
& \leq C\left(\int_{0}^{t}\|v(s)\|^{2}+\varepsilon^{2}\left\|\partial_{t} v(s)\right\|^{2} d s+\varepsilon^{N}\right)
\end{aligned}
$$

thanks to (41), (42), and (9). Note that we have also used that $S A^{0}$ is positive definite symmetric. Thanks to Young's inequality, we have

$$
\varepsilon\left|\left(k A v, \partial_{x} v\right)(t)\right| \leq C\left(\eta\|v(t)\|^{2}+\frac{\varepsilon^{2}}{\eta}\left\|\partial_{x} v(t)\right\|^{2}\right)
$$

for any $\eta>0$. Hence choosing $\eta$ sufficiently small such that $C \eta<1$ and then $\Gamma$ sufficiently large such that $\Gamma>\frac{C}{\eta}$, we finally get

$$
\begin{aligned}
& \|v(t)\|^{2}+\varepsilon^{2}\left\|\partial_{t} v(t)\right\|^{2}+\varepsilon^{2}\left\|\partial_{x} v(t)\right\|^{2} \\
& +\left(\int_{0}^{t} \varepsilon\left\|\partial_{x} v(s)\right\|^{2}+\varepsilon^{3}\left\|\partial_{t x} z(s)\right\|^{2}+\varepsilon^{3}\left\|\partial_{x x} z(s)\right\|^{2} d s\right) \\
& +\alpha\left(|w(t, 0)|^{2}+\varepsilon^{2}\left|\partial_{t} w(t, 0)\right|^{2}\right) \leq C\left(\int_{0}^{t}\|v(s)\|^{2}+\varepsilon^{2}\left\|\partial_{t} v(s)\right\|^{2} d s+\varepsilon^{N}\right) .
\end{aligned}
$$

We obtain a higher-order estimate by using the same scheme of proof. We first come back to (49), but we use an estimate similar to (46) to bound

$$
\frac{1}{\varepsilon} \int_{0}^{+\infty} a\left(\frac{x}{\varepsilon}\right)\left|\partial_{t} v\right|^{2} d x
$$

by

$$
\varepsilon \delta\left\|\partial_{t x} v\right\|^{2}+\delta\left|\partial_{t} w(t, 0)\right|^{2}
$$

Next using Lemma 7, we perform estimates analogous to (49), (57), and (62) for $\partial_{t t} v, \partial_{t x} v$ and $\partial_{t x x} z$ respectively.

Finally, we obtain the estimate

$$
E(t) \leq C\left(\varepsilon^{N}+\int_{0}^{t} E(s) d s\right) .
$$

Therefore, by Gronwall's Lemma we have

$$
E(t) \leq C \varepsilon^{N} \quad \forall t \in\left[0, T^{*}\right] .
$$


Hence $E\left(T^{*}\right)<\varepsilon^{N_{1}}$ since $N>N_{1}$ and hence $T^{*}=T$.

\section{REFERENCES}

[1] M. Gisclon, Étude des conditions aux limites pour un système strictement hyperbolique, via l'approximation parabolique, J. Math. Pures Appl. (9) 75 (1996), no. 5, 485-508. MR 97f:35129

[2] M. Gisclon and D. Serre, Étude des conditions aux limites pour un système strictement hyberbolique via l'approximation parabolique, C. R. Acad. Sci. Paris Sér. I Math. 319 (1994), no. 4, 377-382. MR 95e:35119

[3] J. Goodman, Nonlinear asymptotic stability of viscous shock profiles for conservation laws, Arch. Rational Mech. Anal. 95 (1986), no. 4, 325-344. MR 88b:35127

[4] E. Grenier and O. Guès, Boundary layers for viscous perturbations of noncharacteristic quasilinear hyperbolic problems, J. Differential Equations 143 (1998), no. 1, 110-146. MR 98j:35026

[5] E. Grenier and F. Rousset, Stability of one-dimensional boundary layers by using Green's functions, Comm. Pure Appl. Math. 54 (2001), no. 11, 1343-1385. MR 2003a:35126

[6] S. Kawashima, Systems of a hyperbolic parabolic type with applications to the equations of magnetohydrodynamics, Ph.D. thesis, Kyoto University (1983).

[7] G. Kreiss and H.-O. Kreiss, Stability of systems of viscous conservation laws, Comm. Pure Appl. Math. 51 (1998), no. 11-12, 1397-1424. MR 2000c:35156]

[8] T. T. Li and W. C. Yu, Boundary value problems for quasilinear hyperbolic systems, Duke University Mathematics Department, Durham, N.C., 1985. MR 88g:35115

[9] C. Mascia and K. Zumbrun, Stability of viscous shock profiles for dissipative symmetric hyperbolic-parabolic systems, Preprint (2001).

[10] A. Matsumura and K. Nishihara, Large-time behaviors of solutions to an inflow problem in the half space for a one-dimensional system compressible viscous gas, Commun. Math. Physics 222 (2001), 449-474. MR 2002m:76083

[11] F. Rousset, The boundary conditions coming from the real vanishing viscosity method, Discrete Continuous Dynamical Systems (to appear).

[12] D. Serre and K. Zumbrun, Boundary layer stability in real vanishing viscosity limit, Commun. Math. Phys. 221 (2001), 267-292.

[13] Y. Shizuta and S. Kawashima, Systems of equations of hyperbolic-parabolic type with applications to the discrete Boltzmann equation, Hokkaido Math. J. 14 (1985), no. 2, 249-275. MR 86k:35107

[14] K. Zumbrun, Multidimensional stability of planar viscous shock waves., Advances in the theory of shock waves, Prog. Nonlinear Differential Equations Appl. 47, Birkhaüser, Boston, MA, 307-516 (2001). (English). MR 2002k:35200

[15] K. Zumbrun and P. Howard, Pointwise semigroup methods and stability of viscous shock waves, Indiana Univ. Math. J. 47 (1998), no. 3, 741-871. MR 99m:35157

ENS Lyon, UMPA (UMR 5669 CNRS), 46, allée D'Italie, 69364 Lyon Cedex 07, France E-mail address: frousset@umpa.ens-lyon.fr

Current address: Laboratoire Dieudonné, Université de Nice-Sophia Antipolis, Parc Valrose, 06108 Nice Cedex 02, France

E-mail address: frousset@math.unice.fr 